\title{
Supraglotoplastía asistida con microdebridador en pacientes con síndrome de Pierre Robin y laringomalacia severa
}

\section{Assisted endoscopic microdebrider supraglottoplasty in patients with Pierre Robin syndrome and severe laryngomalacia}

\author{
Diana M. Centeno A¹, Christian Olavarría L², Matías Gómez G .
}

\begin{abstract}
RESUMEN
Se presenta el caso de una paciente recién nacida de término portadora de un síndrome de Pierre Robin asociada a laringomalacia severa que requirió resolución quirúrgica para estabilización de su vía aérea superior. Se presenta la experiencia clínica en el uso de microdebridador para realización de supraglotoplastía como una novedosa alternativa en el tratamiento quirúrgico de este tipo de pacientes y se realiza revisión de la literatura respecto de esta técnica.
\end{abstract}

Palabras claves: Supraglotoplastía, Pierre Robin, laringomalacia severa, microdebridador.

\section{ABSTRACT}

Pierre Robin syndrome is a triad formed by micrognathia, and cleft palate glossoptosis.

His association with laryngomalacia is not set however determining the coexistence of these two diseases and their severity is of utmost importance as they condition the prognosis of a patient with Pierre Robin syndrome.

Currently one of the surgical techniques used in patients with severe laryngomalacia, is assisted endoscopic microdebrider supraglottoplasty. In the ENT unit of the Hospital Luis Calvo Mackenna, this procedure is being implemented in order to give our patients a safe and effective treatment option for their pathology.

Key words: Supraglottoplasty, microdebrider, Pierre robin, laryngomalacia.

\section{INTRODUCCIÓN}

La obstrucción de la vía aérea superior secundaria a micrognatia fue descrita por primera vez por Pierre Robin ${ }^{1}$. El describió una tríada que nominó Secuencia o síndrome de Pierre Robin (SPR) que consiste en la presencia de micrognatia, glosoptosis y, en algunos pacientes, fisura palatina. Se estima una prevalencia de 1:8.500 RN vivos (1:2.000 a 1:30.000), siendo el $80 \%$ asociado con síndromes específicos. La micrognatia puede producir obstrucción de la vía aérea superior debido al colapso posterior de la lengua y a una obstrucción física de las regiones oro e hipofaríngeas 9 .

\footnotetext{
Médico Otorrinolaringología Hospital Calvo Mackenna.

Médico Otorrinolaringología Hospital Clínico José Joaquín Aguirre.

Médico Otorrinolaringología Hospital del Salvador.
} 
La mayoría de los niños que nacen con este problema son manejados mediante posición en decúbito ventral la cual mantiene por gravedad la lengua en una posición más anterior facilitando su respiración y alimentación y, a medida que el niño crece, esta condición mejora por crecimiento de la mandíbula ${ }^{7}$. Sin embargo, algunos debutan con importante dificultad respiratoria y problemas alimenticios con curvas de crecimiento ponderal insuficientes que obligan a tomar decisiones más agresivas como la traqueostomía u otros tipos de procedimientos quirúrgicos ${ }^{2,3}$. Antiguamente, la traqueostomía era la forma de manejo más efectiva y definitiva para estos pacientes ${ }^{4}$, sin embargo se asocia a frecuente morbilidad y ocasionalmente, mortalidad ${ }^{5,6}$.

La distracción mandibular osteogénica (DM0), es un procedimiento opcional y alternativo en niños con SPR que no mejoran con medidas posicionales y que ha demostrado ser efectivo, de baja morbilidad y seguro en el alivio de los síntomas obstructivos de la vía aérea superior mediante el alargamiento gradual de la mandíbula ${ }^{7,10,11}$. Además, se ha estudiado también la calidad de vida (QOL) de los pacientes sometidos a dicho procedimiento encontrándose un resultado subjetivo beneficioso en la población estudiada ${ }^{8}$.

Por otro lado, si bien no encontramos estudios que expresaran la asociación porcentual entre síndrome de Pierre Robin y laringomalacia, el diagnóstico de esta última y la determinación de su severidad, es fundamental para poder pronosticar la evolución que tendrá un paciente con SPR ya que la presencia de una laringomalacia moderada a severa puede contraindicar la realización de una distracción mandibular osteogénica ${ }^{7}$.
A pesar de que la laringomalacia es la causa más común de estridor congénito inspiratorio en neonatos ${ }^{12,20}$, rara vez requiere tratamiento quirúrgico. La mayoría de los pacientes tienen un curso autolimitado y benigno con una resolución espontánea del $90 \%$ entre los 12 y 18 meses de edad ${ }^{13,20}$. Sólo el $10 \%$ al $20 \%$ son catalogados como "severos", y requieren una intervención quirúrgica ${ }^{22,23}$. Su diagnóstico se realiza mediante la historia clínica y nasofibroscopía la que muestra mucosa aritenoidea redundante, acortamiento repliegues ariepiglóticos y/o desplazamiento posterior de la epiglotis $^{21}$.

En 1995, G Roger y cols publicaron un estudio retrospectivo de 115 casos en donde establecen los criterios clínicos para definir a una laringomalacia como "severa" (Tabla 1).

Las opciones quirúrgicas para el manejo de la laringomalacia severa han evolucionado en los últimos 25 años desde la traqueostomía hacia técnicas endoscópicas menos invasivas.

A pesar de que la primera descripción de resección de mucosa supraglótica redundante fue publicada en 1920, no fue hasta 1980 en que este procedimiento se instala como un procedimiento que desplaza a la traqueostomía como primera opción ${ }^{14,16}$.

La supraglotoplastía fue descrita por primera vez por Zalzal y cols en 1987 y es actualmente considerada la técnica de elección para los pacientes con laringomalacia severa con una tasa de éxito que varía entre $38 \%$ a $100 \%{ }^{22}$. O'Donnell y cols, alcanzan según su publicación un éxito del $90 \%$ definido por mejoría del estridor ${ }^{24}$.

La supraglotoplastía está dirigida específicamente a los componentes de la supraglotis que contribuyen al

Tabla 1. Criterios diagnósticos de laringomalacia severa

\begin{tabular}{|l|}
\hline - Disnea en reposo \\
- Disnea severa de esfuerzo con requerimiento de oxígeno \\
- Historitad para alimentarse \\
- Alteración de las curvas pondoestaturales \\
- Apnea/hipoapnea del sueño con polisomnografías alteradas \\
- Reflujo gastroesofágico incontrolable \\
- Hipercapnia
\end{tabular}


colapso de la vía aérea superior incluyendo frecuentemente la resección de la mucosa redundante de los cartílagos aritenoides y las porciones laterales de la epiglotis, y la liberación de los repliegues ariepiglóticos cortos.

A continuación, haremos la descripción de un caso clínico de esta asociación y una modificación quirúrgica para su resolución.

\section{Descripción del caso clínico}

Paciente de sexo femenino nacida por cesárea a las 39 semanas de gestación en el Hospital Regional de Talca con un peso de 3.850 grs. Puntuación de Apgar 9-9.

En el examen físico del recién nacido se objetiva micrognatia, glosoptosis y paladar blando fisurado realizándose el diagnóstico de síndrome de Pierre Robin.

La paciente evoluciona con deficiente incremento ponderal, estridor inspiratorio y dificultad respiratoria que no responde a la administración de oxígeno ni a la aplicación de presión positiva contínua sobre la vía aérea superior (CPAP, en la sigla inglesa) por lo que se decide su traslado a la Unidad de Cuidados Intensivos del Hospital Luis Calvo Mackenna donde la paciente es conectada a ventilación mecánica con un tubo nasotraqueal de 3,5 $\mathrm{mm}$ de diámetro (Figuras 1 y 2).

En dicho hospital, la paciente es evaluada por un equipo multidisciplinario que indica la realización de una distracción ósea previa evaluación inicial en pabellón de la vía aérea en su totalidad, la cual muestra leve traqueomalacia sin otras alteraciones que no contraindica la realización de la distracción mandibular osteogénica (DMO) la que es llevada a cabo sin inconvenientes (Figuras 3 y 4); sin embargo, la paciente evoluciona con gran esfuerzo respiratorio (tiraje costal y subesternal), estridor inspiratorio, y requerimiento de oxígeno por lo que se decide reintubación y nueva evaluación de la vía aérea en pabellón.

En esta segunda oportunidad, la evaluación de la vía aérea es realizada por los autores del trabajo. El procedimiento se realiza primero con nasofibroscopio flexible y luego con endoscopio rígido de $0^{\circ} 4 \mathrm{~mm}$ en ventilación espontánea objetivándose la presencia de

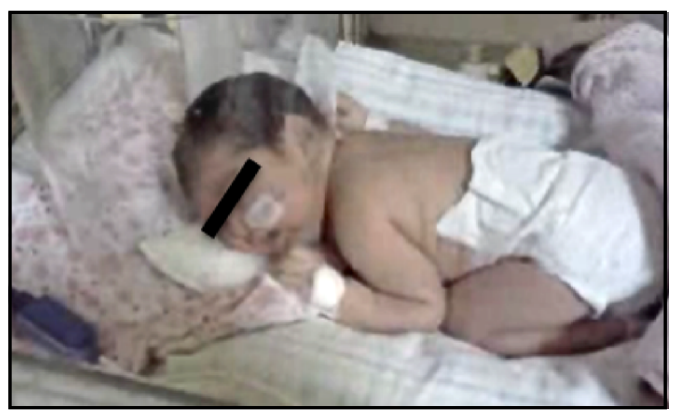

Figura 1.

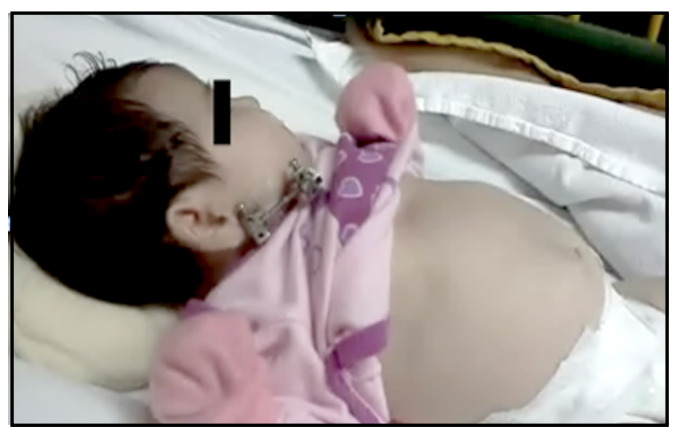

Figura 3.

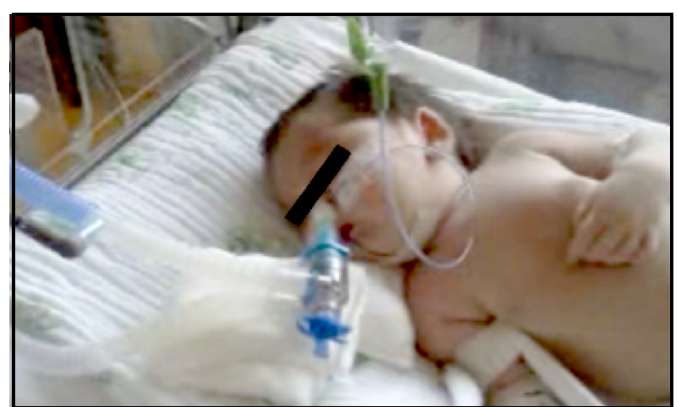

Figura 2.

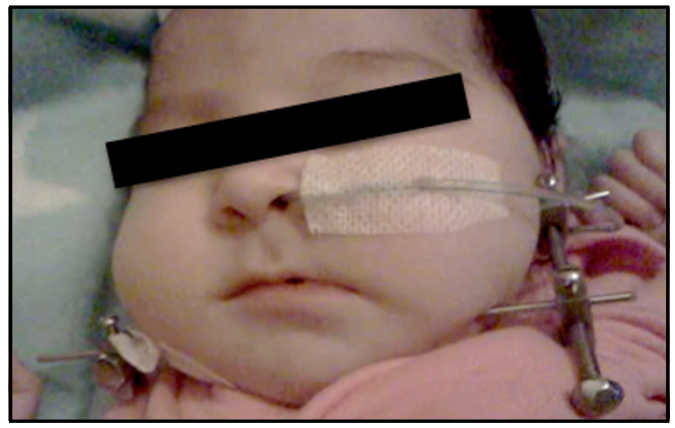

Figura 4. 
una úvula bífida por la fisura palatina y con gran elongación que lleva a la misma al contacto con la glotis. Además, se observa gran edema de aritenoides y pliegues ariepiglóticos cortos. Con cierta dificultad, se logra visualización de glotis con adecuada movilización de cuerdas vocales. Subglotis, tráquea y bronquios dentro de parámetros normales. El diagnóstico de laringomalacia moderada a severa se basó en la clasificación según Daniel R. Olney y cols (Figura 5) ${ }^{26}$.

El caso fue evaluado en equipo multidisciplinario y se decidió la realización de una supraglotoplastía con microdebridador y acortamiento del extremo distal de la úvula con punta de tungsteno. A las 72 horas de la cirugía, se realiza extubación en pabellón bajo visión endoscópica observándose ligero edema en zona intervenida pero vía aérea permeable. La paciente no presenta dificultad respiratoria ni estridor. A los 25 días de operada (50 días de vida), se realiza control en policlínico con nasofibroscopio flexible y se objetiva vía aérea permeable y anatomía laríngea conservada. Se decide retirar sonda nasogástrica. A los 2 meses de vida, se retiran distractores mandibulares con evolución satisfactoria. La paciente presenta un seguimiento de un año y 5 meses de evolución y se encuentra asintomática.

\section{Descripción del procedimiento}

El procedimiento se puede dividir en dos etapas. La primera, llamada diagnóstica, se basa en la visualización endoscópica de la vía aérea en su totalidad para confirmar el diagnóstico de laringomalacia severa y para descartar otras lesiones sincrónicas de la misma que pudieran oscurecer el resultado de la supraglotoplastía. Esta etapa, debe realizarse con el paciente en ventilación espontánea con el objetivo de obtener una imagen dinámica de la vía aérea. En la segunda etapa, el paciente es intubado, en lo posible con el tubo endotraqueal más pequeño que nos permita ventilar adecuadamente al paciente pero a la vez, darnos el mayor espacio para trabajar. A continuación, se realiza la laringoscopía directa. Se introduce el microdebridador a través del laringoscopio en una mano mientras que con la otra se sostiene el endoscopio rígido de $0^{\circ} 4 \mathrm{~mm}$ de diámetro. El microdebridador se utiliza con una punta laríngea de $2,9 \mathrm{~mm}$ en modo oscilante entre 1.500 y 2.500 revoluciones por minuto. Es muy importante

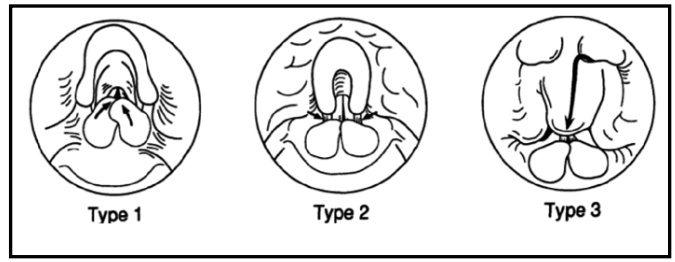

Figura 5.

controlar la presión de succión del aspirador para evitar resecciones excesivas. Las zonas a resecar serán las que generen obstrucción de la vía aérea. En nuestra paciente se realizó resección de mucosa redundante de cartílagos aritenoides y liberación de Ios repliegues ariepiglóticos. El paciente requiere unidad de cuidados intensivos para mejor control de los parámetros ventilatorios y se indican corticoides inhalatorios y tratamiento con inhibidor de bomba de protones. En nuestro caso no se administraron antibióticos.

\section{DISCUSIÓN}

Existen diversas técnicas para llevar a cabo una supraglotoplastía. Tradicionalmente se utilizaban técnicas con bisturí frío o con láser $\mathrm{CO}_{2}$, sin embargo, Zalzal, pionero de la técnica con microdebridador, en una serie de casos en 2005, explica en su publicación cómo se realiza el procedimiento desde la anestesia hasta el procedimiento en sí mismo ${ }^{15}$.

A diferencia de Zalzal quien extuba inmediatamente el paciente una vez finalizado la supraglotoplastía, en nuestro caso, la paciente permaneció intubada por 72 horas por el importante edema que presentaba la laringe. Al cumplirse el plazo antes mencionado, la paciente fue extubada bajo visión endoscópica en pabellón objetivándose vía aérea absolutamente permeable. A la semana de operada, la paciente fue dada de alta sin ningún inconveniente y hasta el día de hoy ha tenido una evolución satisfactoria y un seguimiento regular durante 1 año y 5 meses.

Las potenciales complicaciones de la supraglotoplastía incluyen laringomalacia persistente, estenosis supraglótica, daño del cartílago laríngeo, sinequias, disfagia, aspiración y muerte, sin embargo la frecuencia de presentación de éstas es baja ${ }^{17,24}$. A pesar de las altas tasas de éxito, entre 


\section{Tabla 2. Factores de riesgo de fracaso de supraglotoplastía}

\begin{tabular}{|l|}
\hline - Historia de prematurez (<34 semanas de gestación) \\
- Síndrome genético asociado \\
- Malformaciones craneofaciales (ej. síndrome de Pierre Robin) \\
- Anomalías cardiovasculares \\
- Tesiones sincrónicas de la vía aérea \\
\hline
\end{tabular}

$19 \%$ y $45 \%$ de los pacientes requerirán una revisión del procedimiento original 0 una traqueostomía ${ }^{22}$. Hasta la fecha, hemos realizado supraglotoplastía con microdebridador en 2 pacientes. El segundo paciente presentaba además de síndrome de Pierre Robin y laringomalacia, un síndrome de Stickler que consiste en una alteración sistémica y progresiva del tejido conjuntivo, especialmente del colágeno lo que probablemente colaboró negativamente en la evolución satisfactoria del paciente.

La supraglotoplastía endoscópica con microdebridador es el procedimiento de elección actualmente según muchos autores para pacientes con laringomalacia severa ${ }^{15,18}$, sin embargo hay que tener en cuenta que el resultado puede no ser favorable en presencia de otras alteraciones sincrónicas de la vía aérea 0 comoborbilidades ${ }^{24}$. Los factores de riesgo para el fracaso de una supraglotoplastía se describen a continuación (Tabla 2): historia de prematurez ( $<34$ semanas de gestación), enfermedad neurológica asociada, síndrome genético asociado, malformaciones craneofaciales (ej. síndrome de Pierre Robin), síndrome de Down, anomalías cardiovasculares. Iesiones sincrónicas de la vía aérea y técnica quirúrgica deficiente ${ }^{19,20}$.

A modo de comentarios finales, el síndrome de Pierre Robin consiste en una tríada formada por micrognatia, glosoptosis y fisura palatina.

Su asociación con laringomalacia no está establecida sin embargo la determinación de la coexistencia de estas dos patologías y su severidad es de radical importancia ya que condicionan el pronóstico de un paciente con síndrome de Pierre Robin.

En la actualidad una de las técnicas quirúrgicas más utilizadas en pacientes con laringomalacia severa, es la supraglotoplastía endoscópica asistida con microdebridador, técnica que está implementándose actualmente en nuestro hospital.

Creemos que la supraglotoplastía asistida con microdebridador es un procedimiento técnicamente simple, efectivo, rápido y con bajo índice de complicaciones. Está claro que tenemos que avanzar en nuestra casuística para así poder lograr una correcta curva de aprendizaje y establecer mejor los resultados obtenidos.

\section{BIBLIOGRAFÍA}

1. P Robin. Glossoptosis due to atresia and hypotrophy of the mandible. Am J Dis Child 1934; 48: 54.

2. L Caouette-Laberge, B Bayet, Y Larocoue. The Pierre Robin sequence: review of 125 cases and evolution of treatment modalities. Plast Reconstr Surg 1994; 93: 934-42.

3. RB Schaefer, JA Stadler, AK Gosain. To distract or not to distract: an algorithm for airway management in isolated Pierre Robin sequence. Plast Reconstr Surg 2004; 113: 1113-25.

4. SM Tomaski, GH Zalzal, HM Saal. Airway obstruction in the Pierre Robin Sequence. Laryngoscope 1995; 105: 111-4.

5. It Singer, C Keresmar, G Legris, et al. Developmental sequelae of long-term infant tracheostomy. Dev Med Child Neurol 1989; 31: 224.

6. A Zeitouni, J Manoukian. Tracheostomy in the first year of life. J Otolaryngol 1993; 22: 431.

7. Carmen Gloria Morovic. Manejo actual en síndrome de Pierre Robin. Rev Chil Pediatr 2004; 75(1): 36-42. 
8. Paul Hong, Michael McNeil, Donald B Kearns, Anthony E Magit. Mandibular distraction osteogenesis in children with Pierre Robin sequence: Impact on health-related quality of life. International Journal of Pediatric Otorhinolaryngology 2012; 76: 1159-63.

9. ShPRINTZEn RJ. The implications of the diagnosis of Robin Sequence. Cleft Palate Craniofac J 1993; 29: 205-9.

10. $P$ Hong. A clinical narrative review of mandibular distraction osteogenesis in neonates with Pierre Robin sequence. Int J Pediatr Otorhinolaryngol 2011; 75: 985-91.

11. JD Sidman, D Sampson, B Templeton. Distraction osteogenesis of the mandible for airway obstruction in children. Laryngoscope 2001; 111: $1137-46$.

12. HOLINGER LD. Etiology of stridor in the neonate, infant and child. Ann Otol Rhinol Laryngol 1980; 89: 397-400.

13. Olney DR, Greinwald JH JR, Smith RJH, Bauman NM. Laryngomalacia and its treatment. Laryngoscope 1999; 109: 1770-5.

14. Zalzal GH, Anon JB, Cotton RT. Epiglottoplasty for the treatment of laryngomalacia. Ann Otol Rhinol Laryngol 1987; 96: 72-6.

15. Zalzal GH, Coluins WO. Microdebrider-assisted supraglottoplasty. Int J Pediatr Otorhinolaryngol 2005; 69: 305-9.

16. Seid AB, Park SM, Kearns MJ, Gugenheim S. Laser division of the aryepiglottic folds for severe laryngomalacia. Int J Pediatr Otorhinolaryngol 1985; 10: 153-8.

17. Schroeder JW Jr, Thakkar KH, Poznanovic SA, HoLINGER LD. Aspiration following $\mathrm{CO}_{2}$ laser- assisted supraglottoplasty. Int J Pediatr Otorhinolaryngol 2007; 72: 985-90.

18. Jan C Groblewski, Rahul K Shah, George H Zalzal. Microdebrider-Assisted Supraglottoplasty for Laryngomalacia. Annals of Otology, Rhinology \& Laryngology 2009; 118(8): 592-7.

19. Kristine E Day, Christopher M Discolo, Jeremy D Meier, Bethany J Wolf, Lucinda A Halstead, and DAVID R WHITE. Risk Factors for Supraglottoplasty Failure. Otolaryngology, Head and Neck Surgery 2012; 146: 298.

20. Denoyelle F, Mondain M, Grésillon N, et al. Failures and complications of supraglottoplasty in children. Arch Otolaryngol Head Neck Surg 2003; 129: 1077-80.

21. ThOMPson D. Laryngomalacia: factors that influence disease severity and outcomes of management. Curr Opin Otolaryngol Head Neck Surg 2010; 18: 564-70.

22. Hoff S, Schroeder J JR, Rastatter J, Hollinger L. Supraglottoplasty outcomes in relation to age and comorbid conditions. Int J Pediatr Otorhinolaryngol 2010; 74: 245-9.

23. Avelino M, LiRiano R, FuJita R, et al. Management of laryngomalacia: experience with 22 cases. Braz J Otorhinolaryngol 2005; 71: 330-4.

24. O’Donnell S, Murphy J, Bew S, Knight LC. Aryepiglottoplasty for laryngomalacia: results and recommendations following a case series of 84 . Int J Pediatr Otorhinolaryngol 2007; 71: 1271-5.

25. J Carlos Arancibia. Secuencia de Pierre Robin. Neumol Pediatr 2006; 1(1): 34-6.

26. Daniel R Olney; John H Greinwald, JR., Richard JH Smith, Nancy M Bauman. Laryngomalacia and Its Treatment. Laryngoscope 1999; 109: 1770-6.

Dirección: Cristian Olavarría 